\title{
FUZZY MATHEMATICAL PROGRAMMING APPROACH FOR SOLVING FUZZY LINEAR FRACTIONAL PROGRAMMING PROBLEM
}

\author{
Chinnadurai Veeramani $^{1}$ And Muthukumar Sumathi ${ }^{1}$
}

\begin{abstract}
In this paper, a solution procedure is proposed to solve fuzzy linear fractional programming (FLFP) problem where cost of the objective function, the resources and the technological coefficients are triangular fuzzy numbers. Here, the FLFP problem is transformed into an equivalent deterministic multi-objective linear fractional programming (MOLFP) problem. By using Fuzzy Mathematical programming approach transformed MOLFP problem is reduced single objective linear programming (LP) problem. The proposed procedure illustrated through a numerical example.
\end{abstract}

Keywords. Triangular fuzzy number, Linear programming problem, multi objective linear fractional programming problem, fuzzy mathematical programming.

Mathematics Subject Classification. 90C31, 91A35, 94D05.

\section{INTRODUCTION}

The fields like, financial and corporate planning, production planning, marketing and media selection, university planning and student admissions, health care and hospital planning, etc. often face problems to take decisions that optimize department/equity ratio, profit/cost, inventory/sales, actual cost/standard cost, output/employee, student/cost, nurse/patient ratio etc. The above problems can be solved efficiently through linear fractional programming (LFP) problems. The coefficients of LFP problems are assumed to be exactly known. In practice, the

Received December 29, 2012. Accepted October 11, 2013.

1 Department of Applied Mathematics \& Computational Sciences, PSG College of

Technology, Coimbatore, India. veerasworld@yahoo.com; ramasumathi.psg@gmail.com 
coefficients (some or all) are not exact due to the errors of measurement or vary with market conditions etc. These situations can be modeled efficiently through fuzzy linear fractional programming (FLFP).

Many researchers are investigated different kind of FLFP problems so far. The FLFP problem can be classified into two categories: LFP problem with fuzzy goals and LFP problem with fuzzy coefficients. Most of the FLFP problems can be modeled and solved by fuzzy goal programming approach (see $[1-3,7,9,10,12,13,17]$ ), but very few authors considered FLFP problem where fuzzy coefficients are fuzzy numbers. Mehra et al. [11] proposed a method to compute an $(\alpha, \beta)$ acceptable optimal solution where $\alpha \in[0,1]$ and $\beta \in[0,1]$ is the grade of satisfaction associated with the fuzzy objective function and with the fuzzy constraints, respectively. Pop and Stancu Minasian [14], analyzed a method to solve the fully fuzzified LFP problem, where all the variables and parameters are represented by triangular fuzzy numbers. Most of the work listed above deal with fuzziness either in the constraint inequalities and/or in the aspiration levels of the decision makers. To best of our knowledge, no work has been studied on fuzzy linear fractional programming with fuzzy coefficients. In this paper, we consider the FLFP problem with cost, technological coefficient and resources are triangular fuzzy numbers. First the given FLFP problem is transformed into a deterministic MOLFP problem. This transformation is obtained by using Zadeh extension principle. By using Fuzzy Mathematical programming approach transformed MOLFP problem is reduced single objective linear programming (LP). The rest of our work is organized as follows: In Section 2, we review some concepts of fuzzy numbers. In Section 3, the method of converting LFP problem into LP problem is discussed. The procedure for transforming MOLFP into MOLP problem and Fuzzy Mathematical programming technique is presented in Section 4. In Section 5, the method for solving FLFP problem using Fuzzy Mathematical Programming approach is developed. In Section 6, advantages of the developed method is discussed. The proposed procedure illustrated through a numerical example in Section 6.

\section{SOME BASIC NOTIONS}

In this section, the basic definitions involving fuzzy sets, fuzzy numbers and operations on fuzzy numbers are outlined.

Definition 2.1. If $\mathrm{X}$ is a collection of objects denoted generically by $\mathrm{x}$, then a fuzzy set $\tilde{A}$ in $X$ is a set of ordered pairs

$$
\tilde{A}=\left\{\left(x, \mu_{\tilde{A}}(x)\right) \mid x \in X\right\}
$$

where $\mu_{\tilde{A}}(x)$ is called membership function or grade of membership(also degree of compatibility or degree of truth) of $x \in \tilde{A}$ that maps $\mathrm{X}$ to $[0,1]$.

Definition 2.2. The support of a fuzzy set $\tilde{A}$ is the set of all points $x$ in $X$ such that $\mu_{\tilde{A}}(x)>0$. 


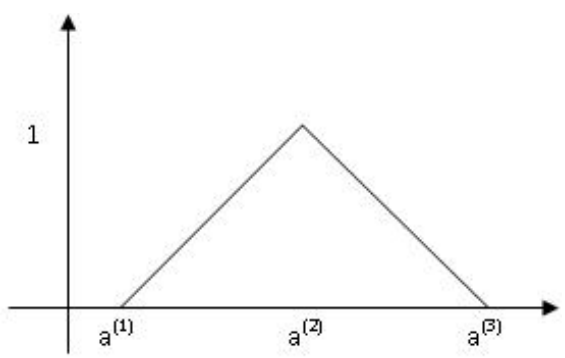

FiguRE 1. Membership function for triangular fuzzy number.

Definition 2.3. A fuzzy set $\tilde{A}$ is said to be normal if $\mu_{\tilde{A}}(x)=1$ for atleast one $x \in X$.

Definition 2.4. A fuzzy set $\tilde{A}$ on $\mathrm{X}$ is convex if and only if $\mu_{\tilde{A}}\left(\lambda x_{1}+(1-\lambda) x_{2}\right) \geq$ $\min \left(\mu_{\tilde{A}}\left(x_{1}\right), \mu_{\tilde{A}}\left(x_{2}\right)\right)$ for all $x_{1}, x_{2} \in X$ and for all $\lambda \in[0,1]$ where min denotes the minimum operator.

Definition 2.5. A fuzzy subset $\tilde{A}$ of the real line $R$ with membership function $\mu_{\tilde{A}}: R \rightarrow[0,1]$ is called fuzzy number if

(i) $\tilde{A}$ is normal and convex fuzzy set.

(ii) Support of $\tilde{A}$ must be bounded.

Any fuzzy number can be described with the following membership function

$$
\mu_{\tilde{A}}(x)=\left\{\begin{array}{c}
L_{\tilde{A}}(x), a \leq x \leq b, \\
R_{\tilde{A}}(x), c \leq x \leq d, \\
0 \quad \text { otherwise, }
\end{array}\right.
$$

where $a \leq b \leq c \leq d, L_{\tilde{A}}(x)$ is increasing and left continuous function on $[a, b]$ and $R_{\tilde{A}}(x)$ is decreasing and right continuous function on $[c, d]$.

Definition 2.6. A triangular fuzzy number $\tilde{A}$ is denoted by $\tilde{A}=\left(a^{(1)}, a^{(2)}, a^{(3)}\right)$ with $a^{(1)}<a^{(2)}<a^{(3)}$ is a fuzzy set where the membership function can be defined as

$$
\mu_{\tilde{A}}(x)=\left\{\begin{array}{cc}
\frac{x-a^{(1)}}{a^{(2)}-a^{(1)}}, a^{(1)} \leq x \leq a^{(2)}, \\
\frac{a^{(3)}-x}{a^{(3)}-a^{(2)}}, a^{(2)} \leq x \leq a^{(3)} \\
0 & \text { otherwise }
\end{array}\right.
$$

whose membership function is shown in Figure 1. 
Definition 2.7. Let ' $*$ ' denote any of the four basic arithmetic operations and $\tilde{A}$, and $\tilde{B}$ denote any two fuzzy numbers. Then, the arithmetic operations through extension principal is defined as

$$
\mu_{\tilde{A} * \tilde{B}}(Z)=\bigvee_{Z=x * y}\left(\mu_{\tilde{A}}(x), \mu_{\tilde{B}}(y)\right) .
$$

Definition 2.8. Let $\tilde{A}=\left(a^{(1)}, a^{(2)}, a^{(3)}\right)$ and $\tilde{B}=\left(b^{(1)}, b^{(2)}, b^{(3)}\right)$ be two positive triangular fuzzy numbers, where $a^{(1)}, a^{(2)}, a^{(3)}, b^{(1)}, b^{(2)}, b^{(3)} \in R$. Then the arithmetic operations and scalar multiplications are defined by

(i) $\tilde{A}+\tilde{B}=\left(a^{(1)}+b^{(1)}, a^{(2)}+b^{(2)}, a^{(3)}+b^{(3)}\right)$;

(ii) $\tilde{A}-\tilde{B}=\left(a^{(1)}-b^{(3)}, a^{(2)}-b^{(2)}, a^{(3)}-b^{(1)}\right)$;

(iii) $K \tilde{A}=\left(K a^{(1)}, K a^{(2)}, K a^{(3)}\right)$, if $\mathrm{K}$ is positive $=\left(K a^{(1)}, K a^{(3)}, K a^{(2)}\right)$, if $\mathrm{K}$ is negative.

Remark 2.9. Let $\tilde{A}=\left(a^{(1)}, a^{(2)}, a^{(3)}\right)$ and $\tilde{B}=\left(b^{(1)}, b^{(2)}, b^{(3)}\right)$ be any two positive triangular fuzzy numbers. If $\tilde{A} \leq \tilde{B}$ then $a^{(2)} \leq b^{(2)}, a^{(1)} \leq b^{(1)}$ and $a^{(3)} \leq b^{(3)}$.

\section{LinEAR FRACTIONAL PROGRAMMing PROBLEM}

In this section, the general form of LFP problem is discussed. Also, Charnes and Cooper's [5] linear transformation is summarized.

The linear fractional programming problem can be written as

$$
\begin{aligned}
& \quad \operatorname{Max} \quad Z(x)=\frac{\sum c_{j} x_{j}+p}{\sum d_{j} x_{j}+q}=\frac{N(x)}{D(x)} \\
& \text { subject to } \begin{array}{r}
x \in S=\left\{x \in R^{n}: A x \leq b, x \geq 0\right\}
\end{array}
\end{aligned}
$$

where $j=1,2, \ldots, n, A \in R^{m \times n}, b \in R^{m}, c, d \in R^{n}$ and $p, q \in R$. For some values of $x, D(x)$ may be equal to zero. To avoid such cases, one requires that either $\{x \geq 0, A x \leq b \Rightarrow D(x)>0\}$ or $\{x \geq 0, A x \leq b \Rightarrow D(x)<0\}$. For convenience, assume that LFP satisfies the condition that

$$
x \geq 0, A x \leq b \Rightarrow D(x)>0 .
$$

Remark 3.1. The problem (1) is said to be standard concave-convex programming problem, if $N(x)$ is concave on $S$ with $N(\zeta) \geq 0$ for some $\zeta \in S$ and $D(x)$ is convex and positive on $S$.

Definition 3.2. [6] The two mathematical programming problem (i) Max $F(x)$, subject to $x \in S$, (ii) Max $G(x)$, subject to $x \in U$ will be said to be equivalent iff there is a one to one map $f$ of the feasible set of (i), onto the feasible set of (ii), such that $F(x)=G(f(x))$ for all $x \in S$. 
Theorem 3.3. Assume that no point $(z, 0)$ with $z \geq 0$ is feasible for the following linear programming problem

$$
\begin{aligned}
& \operatorname{Max} c^{T} z+p t \\
& \text { subject to } \\
& d^{T} z+q t=1 \\
& A z-b t=0 \\
& t \geq 0, z \geq 0, z \in R^{n}, t \in R
\end{aligned}
$$

Now assume that the condition (2), then the LFP (1) us equivalent to linear program problem (3).

Consider the two related problems

$$
\begin{array}{cc} 
& \operatorname{Max} \quad t N(z / t) \\
\text { subject to } & \\
& A(z / t)-b \leq 0 \\
& t D(z / t)=1 \\
& t>0, z \geq 0
\end{array}
$$

and

$$
\begin{array}{cc} 
& \operatorname{Max} \quad t N(z / t) \\
\text { subject to } & \\
& A(z / t)-b \leq 0 \\
& t D(z / t) \leq 1 \\
& t>0, z \geq 0
\end{array}
$$

where (4) is obtained from (1) by the transformation $t=1 / D(x), z=t x$ and (5) differs from (4) by replacing the equality constraint $t D(z / t)=1$ by an inequality constraint $t D(z / t) \leq 1$.

Theorem 3.4. Let for some $\zeta \in S, N(\zeta) \geq 0$, if (1) reaches a (global) maximum at $x=x^{*}$, then (5) reaches a (global) maximum at a point $(t, z)=\left(t^{*}, z^{*}\right)$, where $z^{*} / t^{*}=x^{*}$ and the objective functions at these points are equal.

Theorem 3.5. If (1) is a standard concave-convex programming problem which reaches a (global) maximum at a point $x^{*}$, then the corresponding transformed problem (5) attains the same maximum value at a point $\left(t^{*}, z^{*}\right)$ where $z^{*} / t^{*}=x^{*}$. Moreover (5) has a concave objective function and a convex feasible set.

Suppose that

$$
\operatorname{Max} Z(x)=\frac{N(x)}{D(x)}
$$

subject to

$$
x \in S=\left\{x \in R^{n}: A x \leq b, x \geq 0\right\}
$$


where $N(x)$ is concave and negative for each $x \in S, D(x)$ is concave and positive on $S$, then

$$
\operatorname{Max}_{x \in S} \frac{N(x)}{D(x)} \Leftrightarrow \operatorname{Min}_{x \in S} \frac{-N(x)}{D(x)} \Leftrightarrow \operatorname{Max}_{x \in S} \frac{D(x)}{-N(x)}
$$

here $-N(x)$ is convex and positive. Therefore, the problem (6) is converted into standard concave-convex programming problem. Hence applying the Theorem (3.1) the problem (6) is transformed to the following linear programming problem:

$$
\begin{array}{ll} 
& \operatorname{Max} \quad t D(z / t) \\
\text { subject to } & \\
& A(z / t)-b \leq 0 \\
& -t N(z / t) \leq 1 \\
& t>0, z \geq 0 .
\end{array}
$$

\section{Multi-objective Linear fractional Programming PROBLEM}

In this section, a procedure for converting MOLFP problem in to MOLP problem is discussed.

The MOLFP problem can be written as follows:

$$
\begin{aligned}
& \qquad \operatorname{Max} \quad Z_{i}(x)=\frac{\sum c_{j} x_{j}+p}{\sum d_{j} x_{j}+q}=\frac{N_{i}(x)}{D_{i}(x)} \\
& \text { subject to } \\
& \qquad x \in S=\left\{x \in R^{n}: A x \leq b, x \geq 0\right\}
\end{aligned}
$$

where $i=1,2, \ldots, k, j=1,2, \ldots, n, A \in R^{m \times n}, b \in R^{m}, c, d \in R^{n}$ and $p, q \in R$.

Mathematical analysis of converting MOLFP problem into MOLP problem is discussed below:

\subsection{Mathematical analysis [4]}

Let, $I$ be the index set such that $I=\left\{i: N_{i}(x) \geq 0\right.$ for some $\left.x \in S\right\}$ and $I^{c}=\left\{i: N_{i}(x)<0\right.$ for some $\left.x \in S\right\}$, where $I \cup I^{c}=\{1,2, \ldots, k\}$. Let $D(x)$ be positive on $S$ where $S$ is non-empty and bounced. Let us take the least value of $1 /\left(d_{i} x+q_{i}\right)$ is $t$ for $i \in I$ and least value of $1 /-\left(c_{i}+p_{i}\right)$ is $t$ for $i \in I^{c}$. That is,

$$
\cap_{i \in I} \frac{1}{d_{i} x+q_{i}}=t \text { and } \cap_{i \in I^{c}} \frac{-1}{c_{i} x+p_{i}}=t
$$

which is equivalent to

$$
\frac{1}{d_{i} x+q_{i}} \geq t \text { for } i \in I \text { and } \frac{-1}{c_{i} x+p_{i}} \geq t, \text { for } i \in I^{c} .
$$


By using the transformation $z=t x(t>0)$, Theorems (3.2) and (3.3) and using (9), MOLFP problem (8) may be written as follows:

$$
\begin{array}{r}
\operatorname{Max} \quad g_{i}(z, t)=\left\{t N_{i}(z / t), \text { for } i \in I ;\right. \\
\left.t D_{i}(z / t), \text { for } i \in I^{c}\right\}
\end{array}
$$

subject to

$$
\begin{gathered}
t D_{i}(z / t) \leq 1, \text { for } i \in I \\
-t N_{i}(z / t) \leq 1, \text { for } i \in I^{c} \\
A(z / t)-b \leq 0, \\
t \geq 0, \quad z \geq 0 .
\end{gathered}
$$

\subsection{Fuzzy mathematical programming approach for SOlving MOLFP}

In an extension of classical linear programming with objective functions represented by fuzzy sets, the complete solution set $(z, t)$ from theoretically well defined membership function expression $\mu_{D}(z, t)=\bigcap_{i=1}^{k} \mu_{i}(z, t)$, Zimmermann [19] proved that, if $\mu_{D}(z, t)$ had a unique maximum value $\mu_{D}\left(z^{*}, t^{*}\right)=\operatorname{Max} \mu_{D}(z, t)$, then $\left(z^{*}, t^{*}\right)$ which is an element of complete solution set $(z, t)$ can be derived by solving a classical linear programming with one variable $\lambda$. If $i \in I$, then membership function of each objective function can be written as

$$
\mu_{i}\left(t N_{i}(z / t)\right)=\left\{\begin{array}{cc}
0, & t N_{i}(z / t) \leq 0 \\
\frac{t N_{i}(z / t)}{Z_{i}}, & 0 \leq t N_{i}(z / t) \leq Z_{i} \\
1, & t N_{i}(z / t) \geq Z_{i}
\end{array}\right.
$$

If $i \in I^{c}$, then membership function of each objective function can be written as

$$
\mu_{i}\left(t D_{i}(z / t)\right)=\left\{\begin{array}{cc}
0, & t D_{i}(z / t) \leq 0 \\
\frac{t D_{i}(z / t)}{Z_{i}}, & 0 \leq t D_{i}(z / t) \leq Z_{i} \\
1, & t D_{i}(z / t) \geq Z_{i}
\end{array}\right.
$$

Using Zimmermann's min operator the model (8) transformed to the crisp model as

$$
\begin{gathered}
\operatorname{Max} \lambda \\
\mu_{i}\left(t N_{i}(z / t)\right) \geq \lambda, \text { for } i \in I \\
\mu_{i}\left(t D_{i}(z / t)\right) \geq \lambda \text { for } i \in I^{c} \\
t D_{i}(z / t) \leq 1, \text { for } i \in I \\
-t N_{i}(z / t) \leq 1, \text { for } i \in I^{c} \\
A(z / t)-b \leq 0, \\
t \geq 0, z \geq 0, \quad 0<\lambda \leq 1 .
\end{gathered}
$$




\section{FuZZY LineAR FRACTIONAL PROGRAMMing PROBLEM}

In this section, a procedure for solving fuzzy linear fractional programming (FLFP) problem where the cost of the objective function, the resources and the technological coefficients are triangular fuzzy numbers, is discussed.

Let us consider the fuzzy linear fractional programming problem

$$
\begin{gathered}
\operatorname{Max} \tilde{Z}=\frac{\sum \tilde{c_{j}} x_{j}+\tilde{p}}{\sum \tilde{d_{j}} x_{j}+\tilde{q}} \\
\text { subject to } \\
\sum \tilde{a_{i j}} x_{j} \leq \tilde{b_{i}}, \quad i=1,2, \ldots, m \\
x_{j} \geq 0, \quad j=1,2, \ldots, n
\end{gathered}
$$

we assume that, $\tilde{c_{j}}, \tilde{p}, \tilde{d_{j}}, \tilde{q}, \tilde{b_{i}}, \tilde{a_{i j}}$ are triangular fuzzy numbers for each $i=1, \ldots, m$ and $j=1, \ldots, n$. Therefore, the problem (14) can be written as

$$
\begin{aligned}
& \operatorname{Max} \tilde{Z}=\frac{\sum\left(c_{j}^{(1)}, c_{j}^{(2)}, c_{j}^{(3)}\right) x_{j}+\left(p^{(1)}, p^{(2)}, p^{(3)}\right)}{\sum\left(d_{j}^{(1)}, d_{j}^{(2)}, d_{j}^{(3)}\right) x_{j}+\left(q^{(1)}, q^{(2)}, q^{(3)}\right)} \\
& \text { subject to } \\
& \sum\left(a_{i j}^{(1)}, a_{i j}^{(2)}, a_{i j}^{(3)}\right) x_{j} \leq\left(b_{i}^{(1)}, b_{i}^{(2)}, b_{i}^{3}\right), i=1,2, \ldots, m \\
& x_{j} \geq 0, \quad j=1,2, \ldots, n .
\end{aligned}
$$

By using Zadeh's extension principle of fuzzy numbers, the problem (15) reduce to an equivalent MOLP problem as follows:

$$
\begin{aligned}
\operatorname{Max} Z_{1} & =\frac{\sum c_{j}^{(1)} x_{j}+p^{(1)}}{\sum d_{j}^{(3)} x_{j}+q^{(3)}} \\
\operatorname{Max} Z_{2} & =\frac{\sum c_{j}^{(2)} x_{j}+p^{(2)}}{\sum d_{j}^{(2)} x_{j}+q^{(2)}} \\
\operatorname{Max} Z_{3} & =\frac{\sum c_{j}^{(3)} x_{j}+p^{(3)}}{\sum d_{j}^{(1)} x_{j}+q^{(1)}}
\end{aligned}
$$

subject to

$$
\begin{array}{ll}
\sum a_{i j}^{(1)} x_{j} \leq b_{i}^{(1)} & \\
\sum a_{i j}^{(2)} x_{j} \leq b_{i}^{(2)} & \\
\sum a_{i j}^{(3)} x_{j} \leq b_{i}^{(3)}, & i=1,2, \ldots, m \\
x_{j} \geq 0, & j=1,2, \ldots, n
\end{array}
$$

which is a MOLFP problem. 
Let us assume that $Z_{1}(x), Z_{2}(x)$ and $Z_{3}(x) \geq 0$ for the feasible region. Hence by using the above procedure (in Sect. 4) the MOLFP problem can be converted into the following MOLP problem.

$$
\begin{aligned}
& \operatorname{Max} g_{1}(z, t)=\sum c_{j}^{(1)} z_{j}+p^{(1)} t \\
& \operatorname{Max} g_{2}(z, t)=\sum c_{j}^{(2)} z_{j}+p^{(2)} t \\
& \operatorname{Max} g_{3}(z, t)=\sum c_{j}^{(3)} z_{j}+p^{(3)} t
\end{aligned}
$$

subject to

$$
\begin{aligned}
& \sum d_{j}^{(3)} z_{j}+q^{(3)} t \leq 1 \\
& \sum d_{j}^{(2)} z_{j}+q^{(2)} t \leq 1 \\
& \sum d_{j}^{(1)} z_{j}+q^{(1)} t \leq 1 \\
& \sum a_{i j}^{(1)} z_{j}-b_{i}^{(1)} t \leq 0 \\
& \sum a_{i j}^{(2)} z_{j}-b_{i}^{(2)} t \leq 0 \\
& \sum a_{i j}^{(3)} z_{j}-b_{i}^{(3)} t \leq 0 \quad i=1,2, \ldots, m \\
& z_{j}, t \geq 0, \quad j=1,2, \ldots, n .
\end{aligned}
$$

Solving the transformed MOLP problem for each objective function we obtain $g_{1}, g_{2}$ and $g_{3}$. Let, $Z_{1}(z, t) \geq g_{1}, Z_{2}(z, t) \geq g_{2}$ and $Z_{3}(z, t) \geq g_{3}$. Using the membership function defined in (11) and (12) the above model reduces the to the crisp model as follows:

$$
\begin{gathered}
\operatorname{Max} \lambda \\
\sum c_{j}^{(1)} z_{j}+p^{(1)} t-Z_{1}^{*} \lambda \geq 0 \\
\sum c_{j}^{(2)} z_{j}+p^{(2)} t-Z_{2}^{*} \lambda \geq 0 \\
\sum c_{j}^{(3)} z_{j}+p^{(3)} t-Z_{3}^{*} \lambda \geq 0 \\
\sum d_{j}^{(3)} z_{j}+q^{(3)} t \leq 1 \\
\sum d_{j}^{(2)} z_{j}+q^{(2)} t \leq 1 \\
\sum d_{j}^{(1)} z_{j}+q^{(1)} t \leq 1 \\
\sum a_{i j}^{(1)} z_{j}-b_{i}^{(1)} t \leq 0 \\
\sum a_{i j}^{(2)} z_{j}-b_{i}^{(2)} t \leq 0 \\
\sum a_{i j}^{(3)} z_{j}-b_{i}^{(3)} t \leq 0 \\
z_{j}, t, \lambda \in(0,1], i=1,2, \ldots, m, j=1,2, \ldots, n .
\end{gathered}
$$




\subsection{Algorithm}

The proposed approach for solving FLFP problem can be summarized as follows:

Step 1. The FLFP problem is converted into MOLFP problem using Zadeh's extension principle of fuzzy numbers.

Step 2. The MOLFP problem is transformed into MOLP problem using the method proposed by Charnes and Cooper [5].

Step 3. Maximize each objective function $Z_{i}(i=1,2,3)$, subject to the given set of constraints. Let $Z_{i}^{*}(i=1,2,3)$, be the maximum value of $Z_{i}$ $(i=1,2,3)$ respectively.

Step 4. Examine the nature of $Z_{i}^{*}(i=1,2,3)$. If $Z_{i}^{*} \geq 0$ (for some $i$ ), then $i \in I$, and if $Z_{i}^{*} \leq 0$ (for some $i$ ), then $i \in I^{c}$.

Step 5. For $i \in I$, then we may assume the maximum aspiration level is $Z_{i}^{*}$ and $i \in I^{c}$, then we may assume the maximum aspiration level is $-1 / Z_{i}^{*}$.

Step 6. Using the membership function defined in (11) and (12) the MOLP problem reduces the to the crisp model.

\section{Advantages of the proposed method}

The existing FLFP models $[1-3,7,9,10,12,13]$ can be used fuzziness either in the constraint inequalities and/or in the aspiration levels of the decision makers. The proposed work has been studied on FLFP model with fuzzy coefficients. The discussed model can be solved using the defuzzification approach. Some times the solution obtained by defuzzification method and proposed approach are same. But in literature there are many defuzzification methods are used. Each one have own merits and demerits. Hence the selecting defuzzification method is a difficult one. Also, we provide the mathematical analysis of the proposed method. Therefore, proposed method is analytically correct. The main advantage of the proposed methodology to solve FLFP problem always yields an efficient solution, reduces the complexity in solving FLFP problem and easy computational.

\section{NumeriCAL EXAMPLE}

Example 7.1. A company manufactures two kinds of products $A$ and $B$ with profit around 5 and around 3 dollar per unit, respectively. However the cost for each one unit of the above products is around 5 and around 2 dollars respectively. It is assume that a fixed cost of around 1 dollar is added to the cost function due to expected duration through the process of production. Suppose the raw material needed for manufacturing product $\mathrm{A}$ and $\mathrm{B}$ is about 3 units per pound and about 5 units per pound respectively, the supply for this raw material is restricted to about 15 pounds. Man-hours per unit for the product $A$ is about $5 \mathrm{~h}$ and product $B$ is about $2 \mathrm{~h}$ per unit for manufacturing but total Man-hour available is about 
10 h daily. Determine how many Products $A$ and $B$ should be manufactured in order to maximize the total profit.

In this case, let $x_{1}$ and $x_{2}$ to be the amount of units of $A$ and $B$ to be produced. Then the above problem can be formulated as:

$$
\begin{aligned}
& \operatorname{Max} Z=\frac{\tilde{5} x_{1}+\tilde{3} x_{2}}{\tilde{5} x_{1}+\tilde{2} x_{2}+\tilde{1}} \\
& \text { subject to } \\
& \begin{array}{c}
\tilde{3} x_{1}+\tilde{5} x_{2} \leq \tilde{15} \\
\tilde{5} x_{1}+\tilde{2} x_{2} \leq \tilde{10} \\
x_{1}, x_{2} \geq 0 .
\end{array}
\end{aligned}
$$

Let us assume that $\tilde{5}=(3,5,7), \tilde{3}=(2,3,4), \tilde{5}=(4,5,6), \tilde{2}=(1,2,3), \tilde{1}=$ $(0,1,2), \tilde{3}=(2,3,4), \tilde{5}=(3,5,7), \tilde{15}=(11,15,19), \tilde{5}=(4,5,6), \tilde{2}=(1,2,3)$ and $\tilde{10}=(8,10,12)$.

Then the problem (12) can be written as

$$
\begin{gathered}
\operatorname{Max} Z=\frac{(3,5,7) x_{1}+(2,3,4) x_{2}}{(4,5,6) x_{1}+(1,2,3) x_{2}+(0,1,2)} \\
\text { subject to } \begin{array}{c}
(2,3,4) x_{1}+(3,5,7) x_{2} \leq(11,15,19) \\
(4,5,6) \\
x_{1}+(1,2,3) x_{2} \leq(8,10,12) \\
x_{1}, x_{2} \geq 0 .
\end{array}
\end{gathered}
$$

The above FLFP problem is equivalent to the following MOLFP problem

$$
\begin{array}{ll} 
& \operatorname{Max} Z_{1}=\frac{3 x_{1}+2 x_{2}}{6 x_{1}+3 x_{2}+2} \\
& \operatorname{Max} Z_{2}=\frac{5 x_{1}+3 x_{2}}{5 x_{1}+2 x_{2}+1} \\
& \operatorname{Max} Z_{3}=\frac{7 x_{1}+4 x_{2}}{4 x_{1}+x_{2}} \\
\text { subject to } \quad & \\
& 2 x_{1}+3 x_{2} \leq 11 \\
& 3 x_{1}+5 x_{2} \leq 15 \\
& 4 x_{1}+7 x_{2} \leq 19 \\
& 4 x_{1}+x_{2} \leq 8 \\
& 5 x_{1}+2 x_{2} \leq 10 \\
& 6 x_{1}+3 x_{2} \leq 12 \\
& x_{1}, x_{2} \geq 0 .
\end{array}
$$


Here, it is observed that $Z_{1}(x), Z_{2}(x)$ and $Z_{3}(x) \geq 0$ for the feasible region. The above MOLFP problem is equivalent to the following MOLP problem

$$
\begin{aligned}
\operatorname{Max}\left\{g_{1}(z, t)\right. & =3 z_{1}+2 z_{2} \\
g_{2}(z, t) & =5 z_{1}+3 z_{2} \\
g_{3}(z, t) & \left.=7 z_{1}+4 z_{2}\right\}
\end{aligned}
$$

subject to

$$
\begin{gathered}
6 z_{1}+3 z_{2}+2 t \leq 1 \\
5 z_{1}+2 z_{2}+t \leq 1 \\
4 z_{1}+z_{2} \leq 1 \\
2 z_{1}+3 z_{2}-11 t \leq 0 \\
3 z_{1}+5 z_{2}-15 t \leq 0 \\
4 z_{1}+7 z_{2}-19 t \leq 0 \\
4 z_{1}+z_{2}-8 t \leq 0 \\
5 z_{1}+2 z_{2}-10 t \leq 0 \\
6 z_{1}+3 z_{2}-12 t \leq 0 \\
z_{1}, \quad z_{2}, \quad t \geq 0
\end{gathered}
$$

If the MOLFP problem is solved for each of the objective function one by one. Let $Z_{1}(z, t) \geq 27 / 50, Z_{2}(z, t) \geq 5 / 4$ and $Z_{3}(z, t) \geq 27 / 25$.

Using the membership function defined in (11) and (12) the above model reduces to the linear programming problem as follows:

$$
\begin{gathered}
\operatorname{Max} \lambda \\
150 z_{1}+100 z_{2}-27 \lambda \geq 0 \\
25 z_{1}+15 z_{2}-4 \lambda \geq 0 \\
175 z_{1}+100 z_{2}-27 \lambda \geq 0 \\
6 z_{1}+3 z_{2}+2 t \leq 1 \\
5 z_{1}+2 z_{2}+t \leq 1 \\
4 z_{1}+z_{2} \leq 1 \\
2 z_{1}+3 z_{2}-11 t \leq 0 \\
3 z_{1}+5 z_{2}-15 t \leq 0 \\
4 z_{1}+7 z_{2}-19 t \leq 0 \\
4 z_{1}+z_{2}-8 t \leq 0 \\
5 z_{1}+2 z_{2}-10 t \leq 0 \\
6 z_{1}+3 z_{2}-12 t \leq 0 \\
z_{1}, \quad z_{2}, \quad t \geq 0, \lambda \in(0,1] .
\end{gathered}
$$

The problem is solved and the solution of the above problem is $z_{1}=0, z_{2}=0.27$, $t=0.1$ and $\lambda=0.99$. Hence the solution of the original problem is $x_{1}=0, x_{2}=2.7$ 


\section{Concluding REMARKS}

In this paper, a method of solving the FLFP problems, where the cost of the objective function, the resources and the technological coefficients are triangular fuzzy numbers, is proposed. In the proposed method, FLFP problem is transformed to a MOLFP problem and the resultant problem is converted to a LP problem, using Fuzzy Mathematical programming method. An illustrative numerical example is given to justify the proposed theory.

Some of the possible future directions are given as follows: A stochastic approach of the above problem can be studied and the comparison between the approaches can be carried out. Further the proposed approach can be extended for solving linear fractional programming problems, where the cost of the objective function, the resources and the technological coefficients are trapezoidal fuzzy numbers or non linear membership functions and solving fuzzy multi-objective linear fractional programming problems.

Acknowledgements. The authors would like to thank anonymous referees for the constructive suggestions that has improved both the quality and clarity of the paper.

\section{REFERENCES}

[1] M. Abousina and A.I. Baky, Fuzzy goal programming procedure to bilevel multi-objective linear fractional programming problems. Int. J. Math. Math. Sci. (2010) (Article ID 148975).

[2] G.R. Bitran and A.G. Novaes, Linear programming with a fractional objective function. Oper. Res. 21 (1973) 22-29.

[3] I.A. Baky, Solving multi-level multi-objective linear programming problemsthrough fuzzy goal programming approach. Appl. Math. Model. 34 (2010) 2377-2387.

[4] M. Chakraborty and S. Gupta, Fuzzy mathematical programming for multi objective linear fractional programming problem. Fuzzy Sets System 125 (2002) 335-342.

[5] A. Charnes and W.W. Cooper, Programming with linear fractional functions. Naval Res. Logist. Quart. 9 (1962) 81-186.

[6] Craven, Fractional Programming. Heldermann verlag, Berlin (1988).

[7] D. Dutta, J.R. Rao and R.N. Tiwari, Effect of tolerance in fuzzy linear fractional programming. Fuzzy Sets Systems 55 (1993) 133-142.

[8] S. Jain, A. Mangal, and P.R. Parihar, Solution of fuzzy linear fractional programming problem. OPSEARCH 48 (2011) 129-135.

[9] D.F. Li and S. Chen, A fuzzy programming approach to fuzzy linear fractional programming with fuzzy coefficients. J. Fuzzy Math. 4 (1996) 829-834.

[10] M.K. Luhandjula, Fuzzy approaches for multiple objective linear fractional optimization. Fuzzy Sets System 13 (1984) 11-23.

[11] A. Mehra, S. Chandra and C.R. Bector, Acceptable optimality in linear fractional programming with fuzzy coefficients. Fuzzy Optimization Decision Making 6 (2007) 5-16.

[12] B.B. Pal and I. Basu, A goal programming method for solving fractional programming problems via dynamic programming. Optim. A J. Math. Program. Oper. Res. 35 (1995) $145-157$.

[13] B.B. Pal, B.N. Moitra and U. Maulik, A goal programming procedure for fuzzy multiobjective linear fractional programming problem. Fuzzy Sets and Systems 139 (2003) 395-405.

[14] B. Pop and I.M. Stancu-Minasian, A method of solving fully fuzzified linear fractional programming problems. J.t Appl. Math. Comput. 27 (2008) 227-242. 
[15] S. Schaible, Fractional programming I: duality. Manage. Sci. A 22 (1976) 658-667.

[16] B. Stanojevi and M. Stanojevi, Solving Method for Linear Fractional Optimization Problem with Fuzzy Coefficients in the Objective Function. Int. J. Comput. Commun. Control 8 (2013) $136-145$.

[17] C. Veeramani, C. Duraisamy and A. Nagoorgani, Solving Fuzzy Multi-Objective Linear Programming Problems with Linear Membership Functions. Australian J. Basic and Appl. Sci. 5 (2011) 1163-1171.

[18] H.J. Zimmermann, Description and optimization of fuzzy systems. Int. J. General Systems 2 (1976) 209-215.

[19] H.J. Zimmermann, Fuzzy Set Theory and its Applications. Kluwer Academic, Boston (1985). 